\title{
Connection behaviour and the robustness of steel-framed structures in fire
}

\author{
Ian Burgess \\ University of Sheffield, Department of Civil \& Structural Engineering, Sheffield S1 3JD, United Kingdom
}

\begin{abstract}
The full-scale fire tests at Cardington in the 1990s, and the collapse of at least one of the WTC buildings in 2001, illustrated that connections are potentially the most vulnerable parts of a structure in fire. Fracture of connections causes structural discontinuities and reduces the robustness provided by alternative load paths. An understanding of connection performance is essential to the assessment of structural robustness, and so to structural design against progressive collapse. The forces and deformations to which connectionscan be subjected during a fire differ significantly from those assumed in general design. The internal forces $\mathrm{i}$ generally start with moment and shear at ambient temperature, then superposing compression in the initial stages of a fire, which finally changes to catenary tension at high temperatures. If a connection does not have sufficient resistance or ductility to accommodate simultaneous large rotations and normal forces, then connections may fracture, leading to extensive damage or progressive collapse of the structure. Practical assessment of the robustness of steel connections in fire will inevitably rely largely on numerical modelling, but this is unlikely to include general-purpose finite element modelling, because of the complexity of such models. The most promising alternative is the component method, a practical approach which can be included within global three-dimensional frame analysis. The connection is represented by an assembly of individual components with known mechanical properties. Component characterization must include high-deflection elevated-temperature behaviour, and represent it up to fracture.In reality a connection may either be able to regain its stability after the initial fracture of one (or a few) components, or the first failure may trigger a cascade of failures of other components, leading to complete detachment of the supported member. Numerical modelling must be capable of predicting the sequence of failures of components, rather than considering the first loss of stability as signifying building failure. It is necessary to use a dynamic analysis, so that loss of stability and re-stabilization can be tracked, includingthe movements of disengaging members and the loadsharing mechanisms which maintain integrity and stability within the remaining structure, until total collapse occurs.
\end{abstract}

\section{Introduction}

Structural steel connections have been extensively investigated over the past three decades to determine their moment-rotation characteristics. However, the importance of tying capacity had been realized even earlier, since the explosion at Ronan Point [1] in 1968 caused progressive collapse of a large part of the building. The UK structural steelwork design code BS5950 [2] now requires connections to have minimum tying capacities. The UK SCI/BCSA design guidance [3] checks the tying capacity as an isolated action, whereas in reality a combination of tying force, shear force and moment usually exists. For individual bolts, resistance to tying force may be affected by co-existence with other forces. For a complete bolted connection combined actions can prevent a uniform distribution of the resultant tying force between the bolts, causing them to fail sequentially, significantly reducing the tying capacity.

In design for fire resistance, the increasing adoption of performance-based design principles means that structures are now treated integrally in structural fire safety design. Connections, as the key components which tie structural members together, are important in maintaining structural integrity and preventing progressive collapse. Evidence from the collapse of the WTC buildings [4, 5] and full-scale fire tests at Cardington [6] have shown that connections are vulnerable to fracture in fire. Only limited research has been done on the performance of connections at elevated temperatures, most of which has concentrated on endplate connections, and has mainly been confined to moment-rotation behaviour. A further complexity is that interactions between structural members during heating 
cause continuous changes in the forces and moments taken by the connections.

A recent trend in the design of composite floor systems has been to fire-protect beams on the main column grid, while leaving other beams unprotected. In the early stages of a fire (with structure temperatures typically up to $600^{\circ} \mathrm{C}$ ) unprotected beams expand against restraint from surrounding structure and this creates high compressive forces. Protected beams eventually deflect considerably under the combined effect of high steel temperatures and enhanced loading, shed from the unprotected members, and will impose high tying forces on their connections. In non-composite steel construction, the beams deflect considerably at high temperatures and experience catenary tension, which is transferred to the supporting structure through the connections. Tests by Ding [7] showed that connections were subjected to tying forces varying from 0.65 to 1.6 times their shear force at high temperatures.

Even if the connections survive the heating phase of a fire, when beams contract from their distorted state during cooling this imposes higher tensile forces on connections, and many recorded connection failures observed in full-scale testing have occurred during this phase. These tying forces, either at high temperature or during cooling, together with the local forces on components of the connections imposed by very high rotations, can clearly fracture components, triggering disproportionate collapse of the structure. This was clearly seen in partial connection failures at Cardington. The most dramatic example of the effect of restrained thermal expansion of long-span steel beams in recent years was revealed by the NIST report [5] on the collapse of "7 World Trade" on 11 September 2001 in the immediate aftermath of the collapse of the Twin Towers. Thermal expansion of very long steel secondary beams caused the connections of the supporting primary beams to columns to be pushed-off, initiating progressive collapse of the building.

\section{Component modelling of connections}

Figure 1[8] schematically illustrates the layout of components within a component-based element.

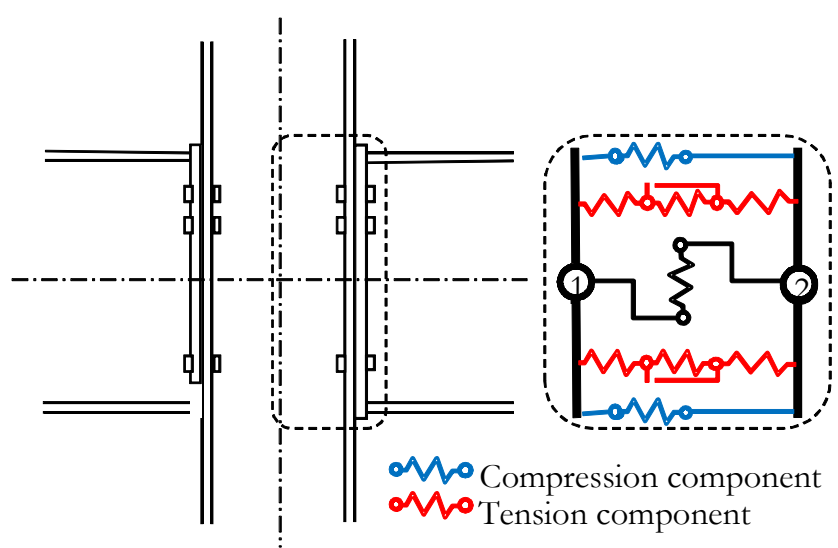

Fig. 1. Component assembly.
The assembled element has 2 external nodes. Although only two component-based "springs" are shown in the figure the model can consist of appropriate numbers of tension (bolt) rows and compression rows. Node 1 is located at the intersection between the beam and column reference axes. Node 2 is the end-node of the beam. Column-face shear components can be included in this assembly, but are commonly assumed to be rigid in the vertical shear direction.

\subsection{Tension components}

Each tension bolt row includes three tension components, which work in series. The middle component in each series is designed to represent the bolt in tension. The other two tension components are: the column flange in tension and end-plate in tension for flush end-plate connections; for other types of connection there will certainly be at least two outer components, although there may be additional effects to be represented by further component springs in each row.

The three components in each tension bolt row are combined into one effective spring at each temperature step, as is illustrated in Figure 2. The force-displacement curves of the tension bolt rows are used to derive the connection's local force and stiffness.
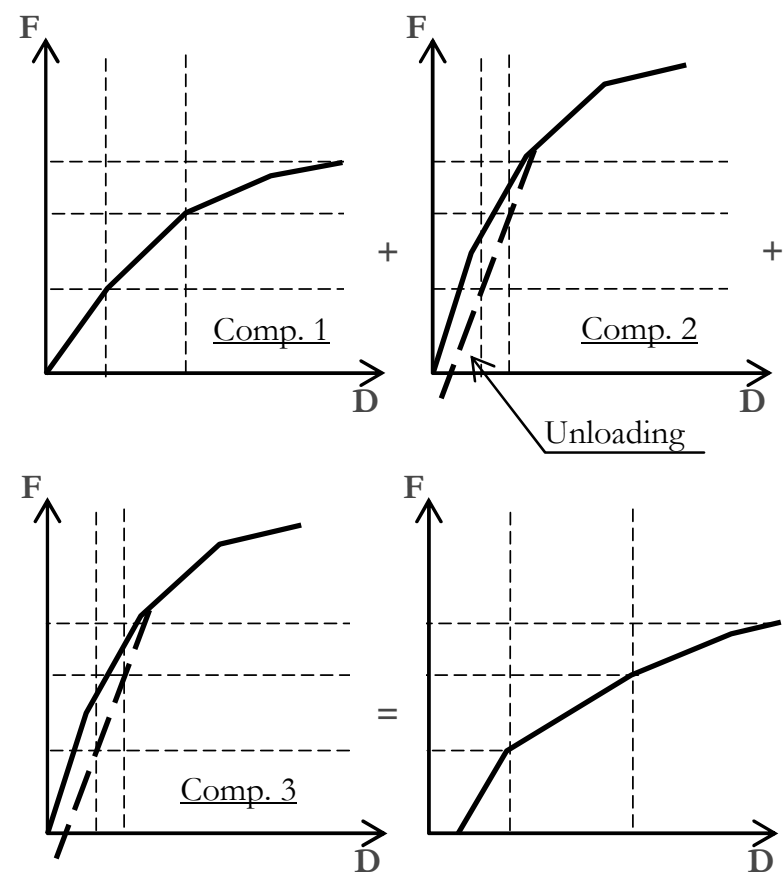

Fig.2.Assembly of the components of a tension bolt row.

After the global analysis reaches a converged stable equilibrium, the forces in the tension bolt rows are established, and the displacements of each tension component are calculated. The related information, such as each component's permanent deformation, is then updated. The maximum resistance of the effective spring is defined by the weakest component in this series. Any force above the weakest component's ultimate resistance is ignored. At each force level, the effective spring's displacement is the total of the components' displacements under this force level. 


\subsection{Compression components}

Compression components clearly do not include bolts, since these are in clearance holes, and become unloaded when the net force at their level is compressive. They may of course include bolts in pure shear in cases where there is a plate connected to the beam web. However, the major effect which needs to be taken into account in the compression zone is hard contact between the beam and column flanges, which may be present at all times (endplate connections) or may occur when the initial clearance between the beam bottom flange and the column-face closes completely. This hard contact generates a stiffness which is much higher than those of the tension components.

As a result of a project conducted by the Universities of Sheffield and Manchester on the capacity and ductility of steel connections at elevated temperatures [9-12] a large number of high-temperature tests were conducted on four common connection types under combined tension and moment. These were accompanied by detailed finite element analyses. The outcome was that many component types were characterized in terms of their load/deformation behaviour. An example of the component arrangement and characteristics (at ambient temperature) is given in Figure 3.
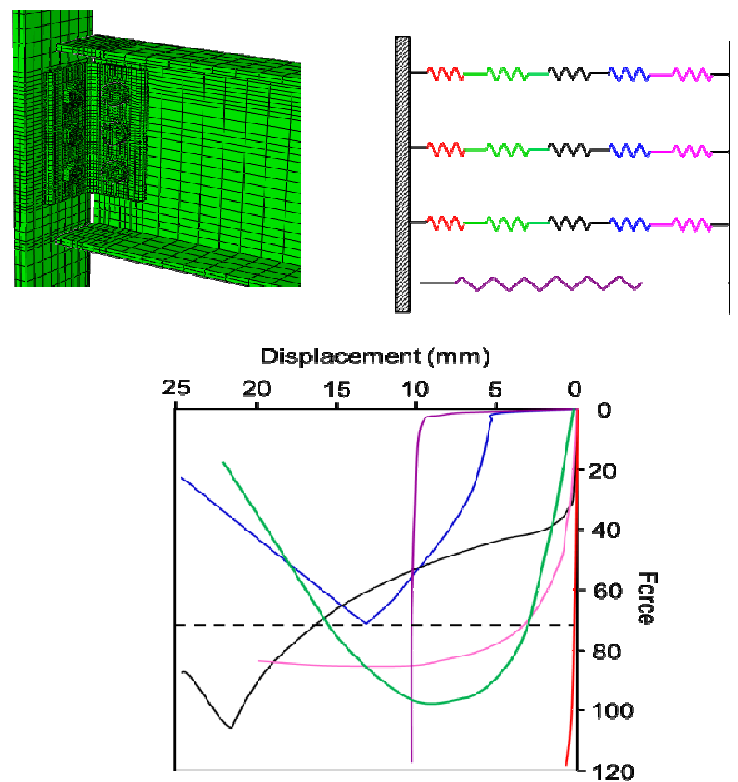

Fig.3.Component characteristics for a web-cleat joint.

\section{Analytical implications}

Because of the nature of conventional quasi-static analysis, an analysis of a structure in fire which includes component-based connection elements can only trace the behaviour of a connection up to the point where its first component fails. In reality a connection may either be able to regain its capacity after the initial fracture of a component, or the first failure may trigger a cascade of failures of other components, leading to complete detachment of the connected member. This possibility should be considered in performance-based design when a structure is being tested for robustness. If connections are to avoid the possibility of becoming detached from members, this numerical modelling must be capable of predicting the sequence of failures of components, rather than simply the first loss of stability. A numerical procedure in which the whole behaviour, from first instability to total collapse, can be modelled effectively, has been developed in Vulcan.

The Vulcan model combines alternate static and dynamic analyses [13], in order to use both to best advantage. Static analysis is used to follow the behaviour of the structure at changing temperature until instability happens; beyond this point an explicit dynamic procedure is activated to track the motion of the system until stability is regained. When combined with the parallel development of general component-based connection elements, this procedure can effectively track the behaviour of connections, from the initial fracture of a component, via the failure of successive bolt-rows, to final detachment from the column. This sequence is illustrated schematically in Figure 4(a); static analysis is initiated at the start of a loading process, and this continues until a loss of stability is detected. The analysis then switches to explicit dynamic, so that the dynamic motion of the unstable structure is tracked until re-stabilization occurs; the static analysis is then restarted. This is capable of modelling the partial fractures of partial-depth end-plates, of the kind shown in Figure 4(b), which were seen in cooling of some Cardington tests.

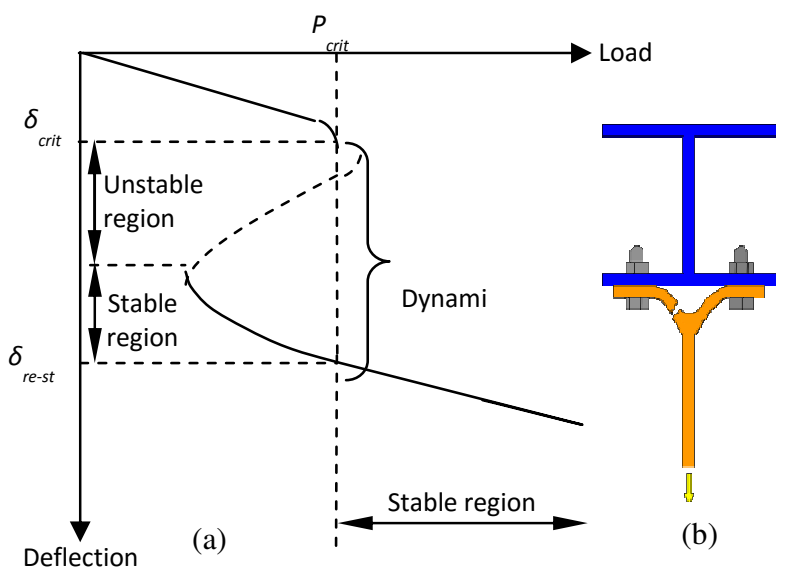

Fig.4.Component characteristics for a web-cleat joint. 
In fact the analysis of a simple frame model, depicted as a sequence of deflected shapes in Figure 5, carries on beyond connection fracture row-by-row, within which the actual fractures in each row were of the components representing the bolts. Logically, this occurs sequentially, starting at the top bolt row and progressing downwards rapidly; the final bolt row essentially simply carries the catenary tension without any of the leverage that applies to the upper rows, and so its curve differs from the others. Detachment of the heated beam does not, in fact cause structural collapse; this occurs due to column buckling at a higher temperature.

\section{The potential of ductile connections}

It seems unlikely that the problem of robustness in fire can be solved using the normal solution of increasing the strength of the vulnerable structural elements, in this case the connections.

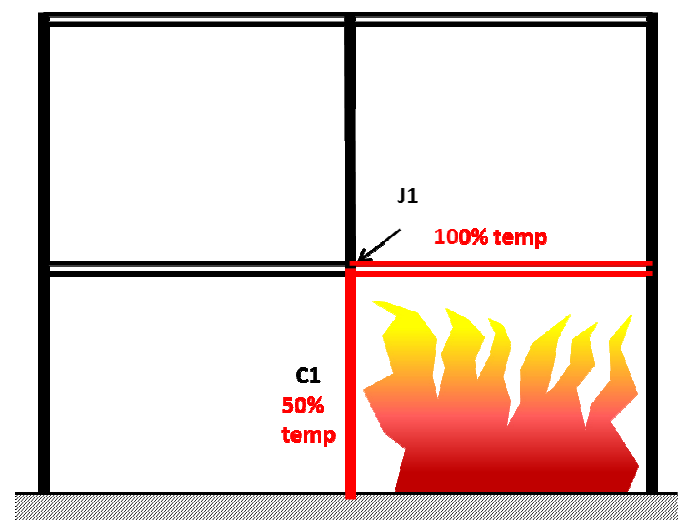

(a)

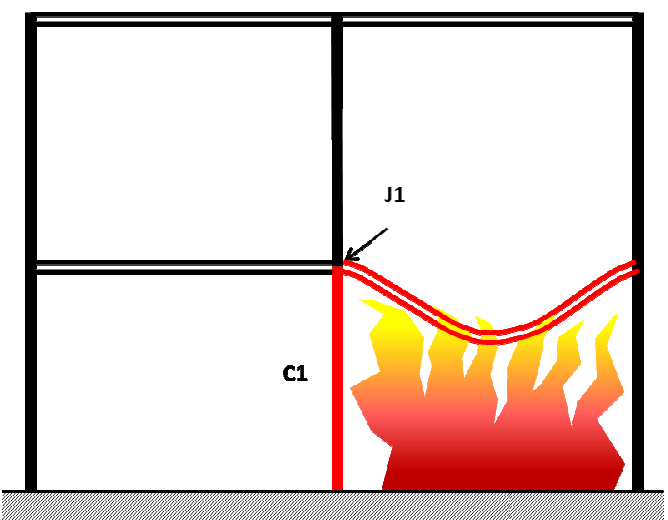

(b)

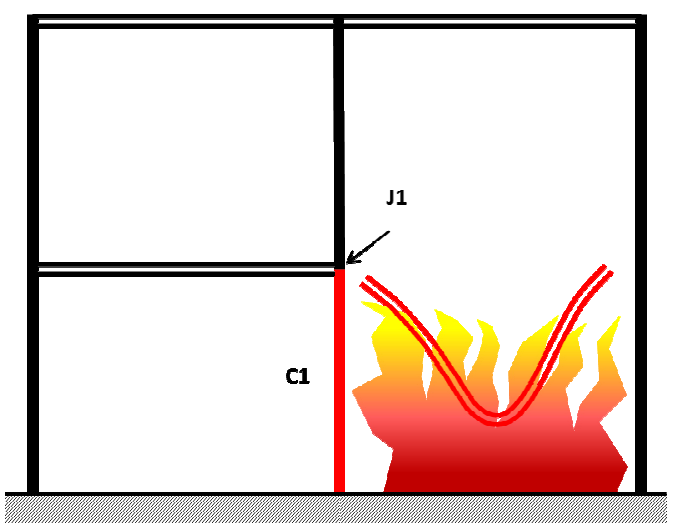

(c)

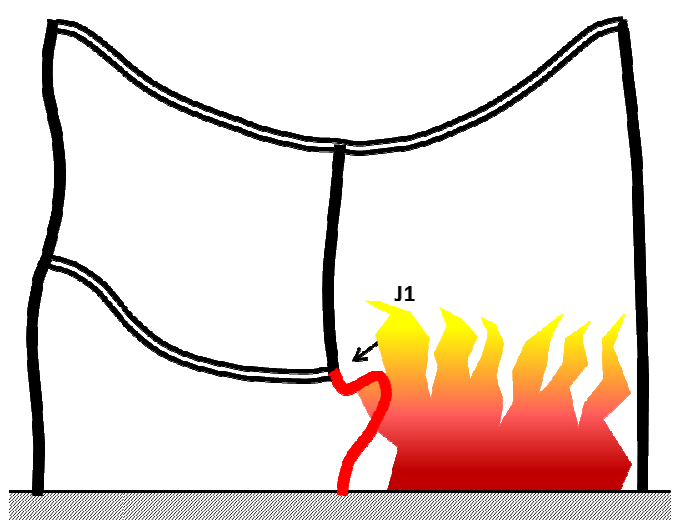

(d)

Fig. 5.Plane frame fire scenario: (a) Initial heating stage, (b) as connection components fracture,(c) connections detached, (d) final collapse due to column buckling. 
The variations of component forces at each bolt row of the connection $\mathrm{J} 1$, and the vertical movement of the central column, with the beam temperature, are plotted in Figures 6(a) and 6(b) respectively.
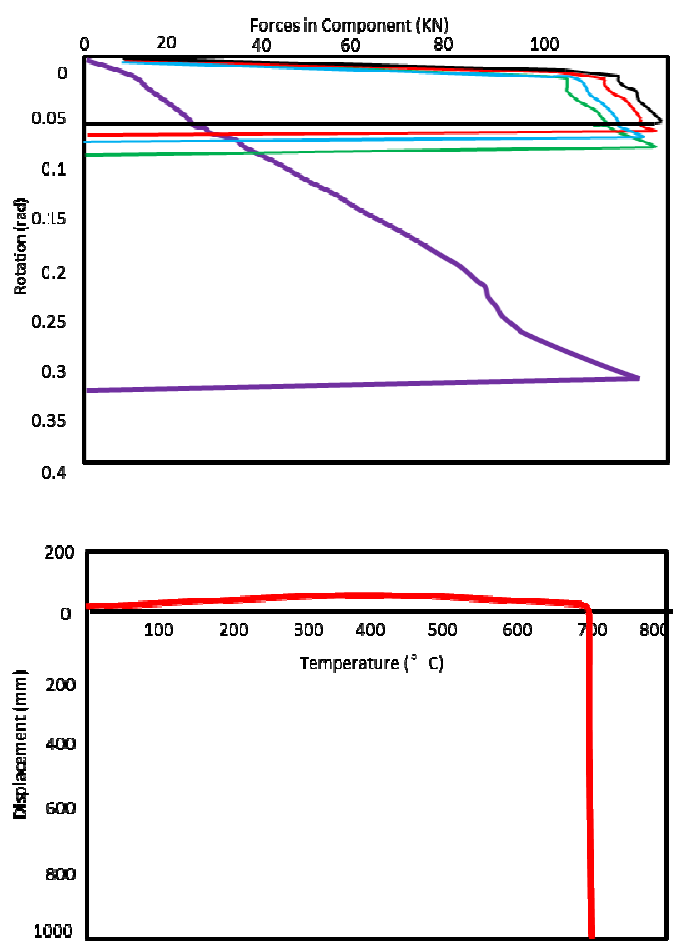

Fig. 6. Plane frame fire scenario: variation of (a) tensile forces in bolt rows of connection, (b) column vertical displacement, with key temperature.

Long-span beams cannot be prevented from generating either large axial expansions or high axial forces in fire, depending on the extent of their restraint from surrounding structure. At relatively low temperatures restrained expansion forces can cause integrity failures in concrete slabs, while at high temperatures the catenary tension forces in beams have magnitudes which are inversely dependent on the amount of axial movement of the beam ends. The latter can even fracture the heated beam in pure tension when its tensile strength is reduced to a few percent of its normal value.

A potential strategy to improve the robustness of connections against fire is to devise connection details which possess the ductility to deform in push-pull without fracturing, while also remaining rigid with respect to vertical shear deformation. In the early stages of heating this will prevent large forces from being transmitted to the surrounding structure. At high temperatures it will allow sufficient movement of the beam ends to reduce the catenary tension in the beam (and hence on the connection) to a level where no fracture occurs at the highest beam temperature. The exact levels of movement required constitute the "ductility demand" of the structure in the fire limit state. The ductility demand is very largely dictated by the characteristics of the beams which are supported by the connections. If the connection is considered as an additional element attached between the beam-end and the column-face then the key demands for a non- composite steel beam are illustrated in Figures 7(a) and 7(b). During the beam expansion phase the ideal situation occurs when the maximum movement at the beam-ends does not cause hard contact with the column faces. In the contraction phase the net movement should be sufficient to reduce the catenary tension below that which causes connection fracture at the highest elevated temperatures. There is a secondary requirement that the additional tension caused by cooling of the beam can be sustained at all stages as the strengths of components are restored
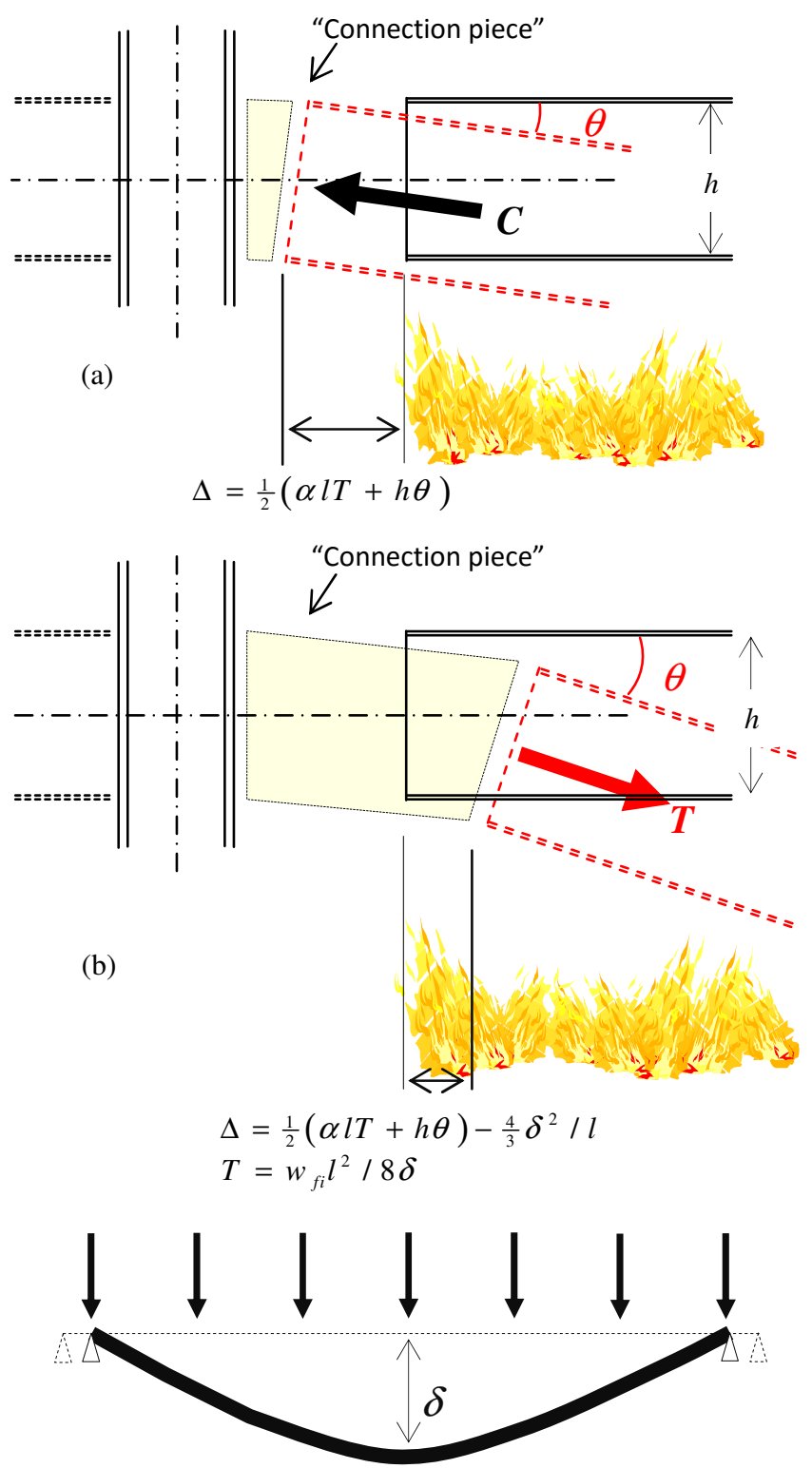

Fig. 7. Movements of beam-end at different stages of heating: (a) Expansion at low temperatures, (b) Shortening due to large deflection, (c) Deflected shape.

\section{Conclusions}

Connections within a structural sub-frame, if heated together with the beams that they support, will initially be subjected to compressive force due to the restrained thermal expansion of the beams. The magnitude of this 
force depends on the span of the beam and the axial restraint stiffness provided by the connections themselves and adjacent structure. Some connections, such as fin plates, can fail due to this force. Although this has been suggested as the cause of failure of WTC 7 [5], it has never been observed in the UK, probably because the multi-storey composite-framed structures typical of UK practice provide less axial restraint to beams. End-plate connections cannot fail under compressive forces; the compression will continue to increase until the beam reaches its limit capacity under the combined effect of the bending moment and compression. It then experiences a rapid increase of deflection, which attenuates the compression force to a limiting value as the thermal expansion is accommodated by the deflection. With further temperature increase the progressive reduction of steel strength decreases the compressive force, to the extent that the axial component eventually becomes tensile. This tension increasingly takes over from the bending resistance of the beam in carrying the loads by catenary action. At this stage the upper bound to the tensile force is given by the lower of the reduced strengths, at the appropriate temperatures, of the beam or its connections. This has been demonstrated in smallscale structural frame tests by Ding [7]. Ductile design of connections is important because the catenary force is reduced with increase of the deflection which is allowed by movement at the ends of the beams; some of this movement can be allowed by the connections themselves. The capacities of a connection in terms of moment, tying force and rotation are completely inter-dependent. Both moment and tying capacity are based on the tensile behaviour of each bolt row. The rotation of a connection, in terms of movement of the beam-end relative to the column face, is the most important influence on its strength and ductility. Semi-rigid or rigid connections, which have higher moment resistances, generally have lower rotational capacity than simple connections, which may limit their ability to develop catenary action. It is not necessary to consider these three parameters directly in order to establish the limit state of a connection in fire. In most cases a component-based model can provide a sufficiently accurate and practical solution to the modeling of connections in fire. Previously componentbased models have been developed mainly for end-plate connections at ambient temperature, in order to generate rotational stiffnesses and moment capacities for semirigid frame design. The Sheffield group has now conducted several successive research projects on steel connection behaviour in fire, culminating in the component characterization reported here. The behaviour of most components of the four connection types tested has been represented in simplified high-temperature nonlinear spring models. Because of the need to emphasize the issue of robustness in fire, it is advantageous for these models to have two innovative characteristics:

- A model of the pre-peak part of the loaddisplacement curve for a component is needed, since uniform distribution of displacement to all the bolt rows is unlikely.
- Formation of a yielding mechanism is not necessarily synonymous with fracture. The behaviour of each component up to large deflection or actual fracture is necessary.

Following these basic principles, components have been characterized for use in modelingof four types of connection, and these have been shown to predict the connection behaviour with satisfactory accuracy. A general-purpose component-based connection element has been assembled which can accommodate the appropriate components within its bolt-rows, and this development has been made in parallel with a static/dynamic solution process for the Vulcan software. It has been shown that the behaviour of a structural frame can be modelled throughout the duration of a fire with this combination, so that transient failures of parts of connections do not cause an end to the analysis, and restabilization is shown if it occurs. This kind of analysis will be necessary in future for true performance-based analytical design of framed buildings against fire, so that potential disproportionate collapse can be predicted and the design of the structure, including that of its connections, can be adjusted to reduce this possibility.

\section{References}

[1] Bignell, V., Peters, J., and Pym, C., Catastrophic failures, Open University Press, Milton Keynes, 1977.

[2] BS5950, Structural Use of Steelwork in BuildingPart 1: Code of practice for design-Rolled and welded sections, British Standards Institution, London, 2001.

[3] SCI \& BCSA, Joints in steel connection, Simple connections, The Steel Construction Institute and British Constructional Steelwork Association, UK, 2002.

[4] NIST, Final Report on the Collapse of the World Trade Center Towers, National Institute of Standards and Technology, USA, 2005.

[5] NIST, Final Report on the Collapse of World Trade Center Building 7, U.S. Department of Commerce and National Institute of Standards and Technology, USA, 2008.

[6] Newman, G.,M., Robinson, J.,T., Bailey, C.,G., Fire Safety Design: A New Approach to Multi-Storey SteelFramed Buildings, The Steel Construction Institute, Ascot, 2004.

[7] Ding, J., Behaviour of Restrained Concrete Filled Tubular Columns and Their Joints in Fire, Ph.D Thesis, University of Manchester, UK, 2007.

[8] Block, F.M., Development of a Component-Based Finite Element for Steel Beam-to-Column Connections at Elevated Temperatures, $\mathrm{PhD}$ thesis, University of Sheffield, 2006.

[9] Yu H.X., Burgess I.W., Davison J.B., and Plank R.J., Experimental and Numerical Investigations of the Behaviour of Flush Endplate Connections at Elevated Temperatures, Journal of Structural Engineering, ASCE,137 (1), (2011) pp 80-87.

[10] Hu, Y., Davison, J.B., Burgess, I.W. and Plank, R.J., 'Component Modelling of Flexible End-plate 
Connections in Fire ', International Journal of Steel Structures, 9, (2009) pp 29-38.

[11] Yu, H.X., Burgess, I.W. Davison, J.B., and Plank, R.J., Experimental Investigation of the Behaviour of Fin Plate Connections in Fire, J. Construct. Steel Research, 65, (2009) pp 723-736.

[12] Yu H.X., Burgess I.W., Davison J.B., and Plank R.J., Tying Capacity of Web Cleat Connections in Fire Part 1: Test and Finite Element Simulation, Engineering Structures, 31 (3), (2009) pp 651-663.

[13] Sun, R.R., Huang, Z. and Burgess, I.W., 'Progressive collapse analysis of steel structures under fire conditions', Engineering Structures, 34, (2012), pp 400-413. 